\title{
Cáncer de mama avanzado receptor de estrógeno positivo: Manejo sistémico actual
}

\author{
César Sánchez R. ${ }^{1,2}$, Yasna Valenzuela V. ${ }^{1}$, Alejandra Pérez-Sepúlveda ${ }^{2}$, Alejandra Villarroel P. ${ }^{3}$, \\ Lidia Medina A. ${ }^{1}$, Mauricio Camus A. ${ }^{4}$ y Francisco Acevedo C. ${ }^{1}$
}

'Departamento de Hematología-Oncología Facultad de Medicina. ${ }^{2} \mathrm{CITO}$ Centro de Investigación en Oncología Traslacional. Departamento de Anatomía Patológica, Facultad de Medicina.

${ }^{4}$ Departamento de Cirugía Oncológica, Facultad de Medicina, Pontificia Universidad Católica de Chile. Santiago, Chile.

Recibido el 12 de octubre de 2017, aceptado el 11 de diciembre de 2017

Correspondencia a: Dr. César Sánchez csanchez@med.puc.c

\section{Systemic therapy for estrogen receptor positive advanced breast cancer}

Breast cancer is the leading cause of cancer death in Chilean women. While most patientes are cured, five percent of cases present with advanced disease initially and up to $20-30 \%$ of patients with localized disease may suffer systemic recurrences. The majority of breast neoplasms are dependent on the estrogenic stimulus, hence the deprivation of estrogen is the main therapeutic strategy. Recently, the use of molecular targeted therapies in combination with endocrine therapy has been successful in improving the survival outcomes of advanced breast cancer, with fewer side effects than those produced by conventional chemotherapy. Knowledge of the mechanisms of action of these new therapies, their toxicities, resistance pathways and patient selection to achieve the best therapeutic benefits are relevant aspects in the management of the disease. We present a review of the current state of management of hormone-dependent metastatic breast cancer with emphasis on the use of endocrine therapies combined with molecular therapies. Key words: breast cancer; breast neoplasm; endocrine therapy; advanced breast cancer; estrogen receptor.

\section{Resumen}

El cáncer de mama es la primera causa de muerte por cáncer en mujeres chilenas. Mientras la mayoría de las personas logra curarse de esta enfermedad, un 5\% de los casos se presenta inicialmente con enfermedad avanzada y hasta un $20-30 \%$ de pacientes con enfermedad localizada pueden sufrir recurrencias sistémicas. La mayoría de las neoplasias mamarias son dependientes del estímulo estrogénico, de allí que la deprivación de estrógenos es la principal estrategia terapéutica. Recientemente, el uso de terapias molecularmente dirigidas en combinación con la terapia endocrina ha logrado mejorar los resultados de sobrevida del cáncer de mama avanzado, con menos efectos colaterales que aquellos producidos por la quimioterapia convencional. El conocimiento de los mecanismos de acción de estas nuevas terapias, sus toxicidades, vías de resistencia y selección de pacientes para lograr los mejores beneficios terapéuticos son aspectos relevantes en el manejo de la enfermedad. Presentamos una revisión del estado actual del manejo del cáncer de mama metastásico hormonodependiente con enfásis en el uso de terapias endocrinas combinadas con terapias moleculares.

Palabras clave: cáncer de mama; neoplasias de mama; terapia endocrina; cáncer de mama avanzado; receptor de estrógenos.

\section{Introducción}

El cáncer de mama $(\mathrm{CM})$ es la principal causa de muerte por cáncer en mujeres en todo el mundo ${ }^{1} \mathrm{y}$ es la primera causa de muerte por cáncer en mujeres chilenas ${ }^{2}$. En Chile, la incidencia de CM el año 2012, se estimó en 31-43 por 100.000 habitantes $^{1}$ y 1.436 personas fallecieron por tumores de mama el año $2014^{2}$. Entre el $60-80 \%$ de los casos de CM co- rresponden a tumores hormono-dependientes, también llamados luminales (Tabla 1). En estos casos, la terapia endocrina (TE) es el pilar del tratamiento. A pesar de la eficacia de esta estrategia, $20-30 \%$ de las pacientes van a sufrir eventualmente una recurrencia sistémica y un $5 \%$ se presentarán al diagnóstico con enfermedad en etapa IV ${ }^{3}$. El CM avanzado (CMA) es aún, en general, una enfermedad incurable, y el principal objetivo terapéutico es aminorar los sín- 
tomas y prolongar la sobrevida, manteniendo una buena calidad de vida ${ }^{4}$.

Expondremos aspectos generales del manejo del CMA receptor de estrógenos positivo $(\mathrm{RE}+) \mathrm{y}$, además, una revisión de la evidencia actual acerca de su manejo óptimo, focalizándonos en el uso de terapias moleculares sumadas a la TE, de acuerdo a una revisión de la literatura de los últimos 5 años.

\section{Material y Método}

Junto a una breve revisión narrativa, se revisó la evidencia actual de terapia del CMA RE+; para ello se realizó una búsqueda en PUBMED, con los términos "advanced", "metastatic", "breast cancer" and "Phase III", excluyendo cáncer de mama "HER2 positive" y "triple negative”. La última búsqueda se hizo el 23-03-2017 con los filtros: "clinical trial", "humans" y "published in the last 5 years".

Aplicando estos filtros se obtuvo 67 citaciones, de las cuales 12 publicaciones eran estudios clínicos fase III en pacientes con CMA RE+, utilizando terapia endocrina.

\section{Extracción de datos en artículos obtenidos de la revisión de PUBMED}

Se obtuvo el dato de número de pacientes incluidos en cada estudio, tratamiento y escenario del tratamiento (primera línea, segunda línea), resultados en sobrevida libre de progresión (SLP) y sobrevida global (SG). Estos datos se incluyeron en una tabla resumen.

\section{Tratamiento actual del cáncer de mama en tumores hormono-dependientes: terapias disponibles}

La TE es la opción preferida para el tratamiento del CMA RE+ incluso en presencia de enfermedad visceral; a menos que se demuestre resistencia endocrina o crisis visceral, definida como una disfunción orgánica severa evaluada por signos, síntomas y estudios de laboratorio (ej. linfangitis pulmonar con compromiso respiratorio, mieloptisis con necesidad de transfusión, meningitis carcinomatosa, metástasis hepáticas asociado a signos de falla hepática). Se considera también como crisis visceral la progresión rápida de la enfermedad o la falla de múltiples líneas de $\mathrm{TE}^{4}$. Ello porque la TE requiere mayor tiempo que la quimioterapia (QT) para mostrar su eficacia, tiempo que en pacientes con crisis visceral es limitado. El uso de QT en pacientes sin crisis visceral no ha demostrado mayor beneficio que el uso de TE. Tampoco el uso de QT combinada con TE ha mostrado mayor beneficio que cualquiera de ellas en forma aislada ${ }^{4,5}$.

El estado menopáusico define las opciones de tratamiento para CMA RE+: mientras en mujeres posmenopáusicas, donde la producción de estrógenos (E2) es principalmente extra-ovárica (tejido graso, glándula suprarrenal), se pueden utilizar la mayoría de las TE disponibles; en mujeres premenopáusicas sólo tamoxifeno. En caso de requerir en estas pacientes el uso de otras terapias como fulvestrant o inhibidores de aromatasa (IA), es necesario la supresión de la función ovárica médica o quirúrgica ${ }^{4}$.

Opciones actuales de TE incluyen moduladores selectivos del RE (MSRE), IA y reguladores negativos de la expresión del RE (SERD), como el fulvestrant. Si bien se recomienda el tratamiento secuencial con estos agentes a medida que avanza la enfermedad, son pocos los estudios que comparan los efectos de diferentes secuencias ${ }^{4,6}$. La elección del tipo de TE depende, principalmente, de los siguientes factores: estado menopáusico, uso y respuesta a terapias previas, tiempo libre de enfermedad, toxicidad esperada y biología del tumor (Tabla 2 y Figura 1).

\section{Tratamiento de primera línea}

\section{Moduladores selectivos del receptor de estrógeno (MSRE)}

El tamoxifeno, es el primer MSRE usado en clínica. La tasa de respuesta en pacientes tratados en primera línea es cercana al $35 \%$ y en pacientes con alta expresión de RE y receptores de progesterona (RP) la respuesta llega a ser de hasta un $70 \%{ }^{7}$. Mientras existen otros MSRE, éstos no son utilizados en forma habitual en el manejo del CMA (raloxifeno, tomerifeno) (Tabla 3).

\section{Inhibidores de aromatasa}

Los IA interactúan con la enzima responsable de la producción de estradiol (la aromatasa) en distintos

Tabla 1. Subtipos intrínsecos de cáncer de mama según marcadores inmunohistoquímicos clásicos

\begin{tabular}{|llll}
\hline Luminal A & RE + y RP+ & HER2- & Ki67 $\leq 20 \%$ \\
Luminal B & RE + y RP - o $<20 \%$ & HER2- & Ki67 $>20 \%$ \\
Luminal B HER2 + & RE+ y/o RP+ & HER2 + & Cualquier Ki67 \\
HER2 enriquecido & RE - y RP- & HER2+ & Cualquier Ki67 \\
Triple negativo & RE- y RP- & HER2- & Cualquier Ki67 \\
\hline
\end{tabular}

RE: Receptor de estrógeno. RP: Receptor de progesterona. HER-2: Receptor de factor de crecimiento epidérmico humano tipo 2. KI67: Antígeno de proliferación celular. 
Tabla 2. Tratamiento del cáncer de mama avanzado (CMA) en tumores positivos para receptores hormonales: aspectos relevantes

- Las indicaciones de quimioterapia en pacientes con CMA RE+ incluyen: crisis visceral, baja probabilidad de respuesta a TE: baja expresión de RE, expresión de HER2, progresión tras múltiples líneas de TE previas

- En pacientes posmenopáusicas, vírgenes a tratamiento, el uso de IA solo o en combinación (IA+fulvestrant; IA + palbociclib) como primera opción es una estrategia de elección, dada las mejores tasas de respuesta y leve mejor sobrevida comparado con tamoxifeno

- El uso de IA no esteroidales (anastrozol, letrozol) como terapia inicial se prefiere sobre el uso de IA esteroidales (exemestane) dada la posibilidad, tras la progresión, de usar este último en combinación con everolimus en pacientes con resistencia a TE. Sin embargo, el uso de un IA esteroidal luego de un no esteroidal o viceversa también es una opción aceptada

- La decisión de tratamiento de primera línea para CMA depende del uso de TE previa y del tiempo de recurrencia en relación al término de la TE adyuvante

- En pacientes resistentes a TE los esquemas de combinación con demostración de utilidad en estudios de fase III son everolimus+IA o Fulvestrant + palbociclib u otros inhibidores de kinasas dependientes de ciclinas

RE+: receptor de estrógeno positivo, TE: terapia endocrina, HER2: receptor para el factor de crecimiento epidérmico humano tipo 2, IA: inhibidor de aromatasa.

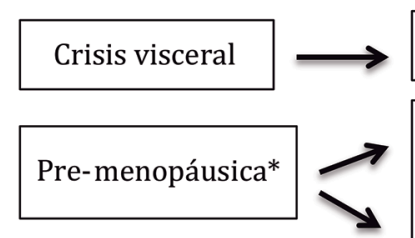

Quimioterapia

Tamoxifeno

Supresión ovarica*

*seguir guías de manejo

en posmenopáusicas

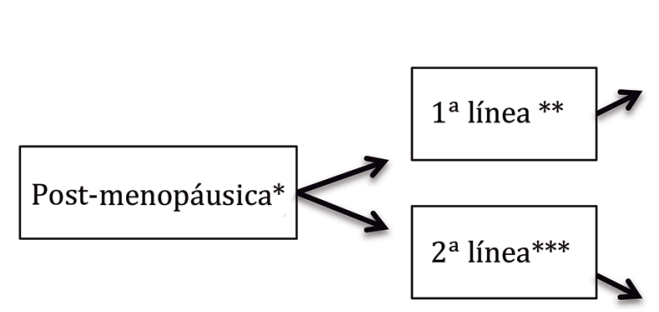

IA

Tamoxifeno

Fulvestrant

Fulvestrant + Palbociclib u otro

inhibidor de $\mathrm{CDK}$

IA (esteroidal) +Everolimus

Estradiol

Progestágenos

Re-exposición a TE previas

Quimioterapia

\begin{abstract}
\& en pacientes con recurrencia precoz tras uso de inhibidor de aromatasa (IA)
* pacientes premenopáusicas que requieran otro tratamiento diferente al tamoxifeno deben convertirse en posmenopáusicas a través de supresión de la función ovárica u ooforectomía, y allí continuar según manejo de mujeres posmenopáusicas

** Primera línea en CMA se define como el primer tratamiento para el CMA, independientemente si la paciente ha recibido tratamiento para su CM con TE en el escenario neoadyuvante o adyuvante con intención curativa. Las opciones de tratamiento se eligen según: terapia previa, por ejemplo, si recae intratratamiento con tamoxifeno, se usa un IA o fulvestrant; el uso de TE combinada con IA + inhibidores de kinasas dependientes de ciclina (CDK) son una opción para lograr mejor respuesta y aumentar la SLP. Aún no existen datos de mejoría en sobrevida global

*** Segunda línea se refiere a pacientes que han sido tratados con TE previamente para el CMA
\end{abstract}

Recaída precoz: antes de 12 meses de término de TE adyuvante Recaída tardía: después de 12 meses desde la TE adyuvante
Figura 1. Algoritmo de manejo para pacientes con cáncer de mama avanzado (CMA) receptor de estrógenos positivo $(\mathrm{RE}+)$. 
Tabla 3. Tipos de terapia endocrina actualmente en uso clínico

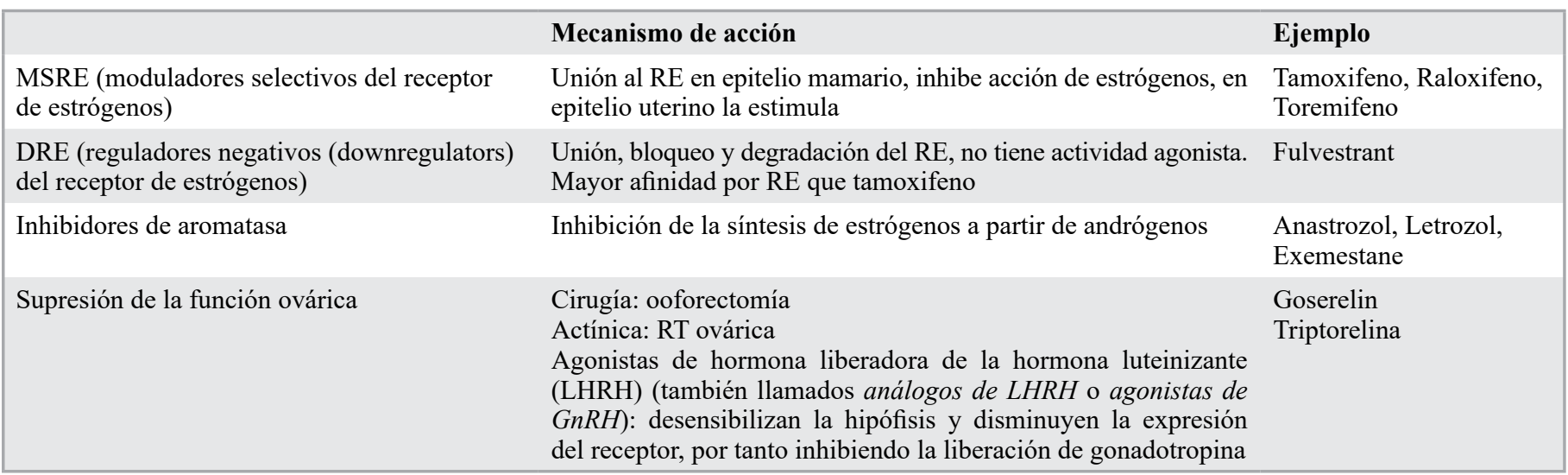

tejidos del organismo. El exemestano es un IA esteroidal que se une irreversiblemente a la aromatasa, mientras que los IA no esteroidales, como el anastrozol y el letrozol, han demostrado unirse reversiblemente a la enzima. Una opción de tratamiento es utilizar un inhibidor no esteroidal tras la progresión con uno esteroidal o viceversa, pero con bajas tasas de respuesta y medianas de tiempo a la progresión de 3 a 5 meses $^{8}$. Actualmente, no existe evidencia clínica que sugiera la existencia de un IA superior a otro aunque sí parecen tener mejor respuestas que el tamoxifeno ${ }^{4}$. Un metaanálisis que incluyó 8.504 pacientes con CMA tratados con IA comparado con tamoxifeno mostró una sobrevida superior para aquellos tratados con IA [hazard ratio (HR) 0,89; intervalo de confianza (IC) del 95\%: 0,80-0,99]'.

\section{Reguladores negativos de la expresión del receptor de estrógeno}

La tercera estrategia terapéutica disponible está dirigida contra la expresión del receptor luego que éste se ha unido a su ligando. El fulvestrant es un antagonista esteroidal del RE, de alta afinidad. El estudio fase $I I \operatorname{FIRST}^{10}(\mathrm{n}=205)$ fue el primer ensayo en sugerir que una TE alternativa podría ser más eficaz que un IA en la primera línea de tratamiento del CMA. Este estudio comparó fulvestrant $500 \mathrm{mg}$ contra $1 \mathrm{mg}$ de anastrozol y mostró beneficio en SG en la rama de fulvestrant: 54 meses versus 48 meses (HR 0,70; IC del 95\%: 0,50-0,98; $\mathrm{p}=0,04)$. El estudio FALCON $3^{11}$ es un estudio fase III que, utilizando las mismas ramas de comparación que el FIRST, mostró un beneficio en la SLP de pacientes con CMA con enfemedad metastásica no visceral, sobre un IA, reforzando la efectividad de fulvestrant en este escenario (Tabla 4). Los pacientes incluidos en este ensayo eran en su mayoría vírgenes a TE para enfermedad avanzada. Este último punto destaca la importancia de la población incluida en los estudios.

Mientras las tasas de respuesta a la deprivación estrogénica, en tumores $\mathrm{RE}+$, pueden llegar a ser de un $80 \%$, éstas son en general de tiempo limitado y no logran curar la enfermedad; por ello se han desarrollado estrategias que superen la resistencia a la TE a través de la combinación de TE o con el uso de terapias biológicas-moleculares en conjunto con la TE.

\section{Terapias endocrinas combinadas}

La aparición de resistencia primaria (de novo) o secundaria (adquirida) a la supresión estrogénica es casi inevitable en pacientes con $\mathrm{CMA}^{12}$. Como los fármacos antes mencionados tienen diferentes mecanismos de acción, la combinación de éstos es un enfoque lógico para mejorar la eficacia de la TE. La combinación de fulvestrant $(250 \mathrm{mg})$ con anastrozol versus anastrozol solo, ha mostrado resultados contradictorios. El estudio $\mathrm{FACT}^{13}$ comparó la combinación de anastrozol con fulvestrant versus anastrozol solo, como segunda línea. Este estudio no mostró ventajas clínicas del uso de la combinación de fármacos (SLP de anastrozol+fulvestrant 10.8 meses versus anastrozol: 10,2 meses; HR 0,99, $\mathrm{p}=0,9137$, SG 38 meses versus 38,2 meses HR $1,0 ; 95 \%$ IC 0,76 a 1,$32 ; p=1,00)$, mientras que el estudio SWOG S0226 mostró beneficio de la combinación de estos fármacos tanto en SLP como en SG, en 707 pacientes con CMA. La SLP fue 13,5 meses en el grupo de monoterapia, y 15 meses en el grupo combinado (HR, $0.80 ; 95 \%$ IC, 0,68 to 0,94 ; $\mathrm{p}=0,007)$. También la SG fue superior en el grupo de terapia combinada (41,3 meses versus 47,7 meses; 
Tabla 4. Resultados de estudios clínicos fase III recientes en cáncer de mama avanzado, luminales. Revisión de la literatura, últimos 5 años

\begin{tabular}{|c|c|c|c|c|c|c|}
\hline Estudio & $\mathbf{n}$ & Ramas & $\begin{array}{l}\text { Selección } \\
\text { pacientes }\end{array}$ & $\begin{array}{l}\text { Objetivo } \\
\text { primario }\end{array}$ & SLP & SG \\
\hline CONFIRM $^{24}$ & 736 & $\begin{array}{l}\text { 1) Fulvestrant } 500 \mathrm{mg} \\
\qquad v \mathrm{~s} \\
\text { 2) Fulvestrant } 250 \mathrm{mg}\end{array}$ & $2^{\mathrm{a}}$ línea & SLP & $\begin{array}{l}\text { Reportado en } \\
\text { trabajo previo }\end{array}$ & $\begin{array}{l}1: 26,4 \text { meses } \\
2: 22,3 \text { meses } \\
p=0,02\end{array}$ \\
\hline SoFEA $^{31}$ & 723 & $\begin{array}{l}\text { 1) Anastrozol + Fulvestrant } \\
\qquad v \\
\text { 2) Fulvestrant } \\
v s \\
\text { 3) Exemestane }\end{array}$ & $1^{\mathrm{a}} / 2^{\mathrm{a}}$ línea & SLP & $\begin{array}{c}1: 4,4 \text { meses } \\
2: 4,8 \text { meses } \\
3: 3,4 \text { meses } \\
\quad \&\end{array}$ & $\begin{array}{l}1: 20 \text { meses } \\
2: 21,6 \text { meses } \\
3: 19,4 \text { meses } \\
\quad \&\end{array}$ \\
\hline BOLERO $2^{25,32}$ & 724 & $\begin{array}{l}\text { 1) Everolimus + Exemestane } \\
\qquad v s \\
\text { 2) Placebo + Exemestane }\end{array}$ & $1^{\mathrm{a}} / 2^{\mathrm{a}}$ línea & SLP & $\begin{array}{l}1: 7,8 \text { meses } \\
2: 3,2 \text { meses } \\
p<0,0001\end{array}$ & $\begin{array}{l}30,98 \text { meses } v s \\
26,55 \text { meses } \\
\&\end{array}$ \\
\hline PALOMA $2^{18}$ & 666 & $\begin{array}{l}\text { 1) Palbociclib+Letrozol } \\
\qquad v s \\
\text { 2) Placebo +Letrozol }\end{array}$ & $1^{\mathrm{a}}$ línea & SLP & $\begin{array}{c}1: 24,8 \text { meses } \\
2: 14,5 \text { meses } \\
p<0,001\end{array}$ & Datos inmaduros \\
\hline MONALEESA $2^{20}$ & 668 & $\begin{array}{l}\text { 1) Ribociclib + Letrozol } \\
\qquad v s \\
\text { 2) Placebo + Letrozol }\end{array}$ & $1^{\mathrm{a}}$ línea & SLP & $\begin{array}{c}1: \text { no alcanzada } \\
2: 14,7 \text { meses } \\
\mathrm{p}=3,29 \text { por } 10^{-6}\end{array}$ & Datos inmaduros \\
\hline $\begin{array}{l}\text { Breast Cancer } 2016 \\
\text { 23:771-934 }\end{array}$ & 222 & $\begin{array}{l}\text { 1) Goserelin 10,8 mg c/3 meses + Tamoxifeno } \\
\qquad v s \\
\text { 2) Goserelin 3,6 mg/mes + Tamoxifeno }\end{array}$ & $1^{\mathrm{a}}$ línea & SLP & $\begin{array}{l}\text { A semana } 24 \\
1: 61,5 \% \\
2: 60,2 \% \\
\&\end{array}$ & No reportada \\
\hline $\begin{array}{l}\text { Breast Cancer Res } \\
\text { Treat 2013; 139: } \\
441-51^{35}\end{array}$ & 298 & $\begin{array}{l}\text { 1) Exemestane } \\
\qquad v s \\
\text { 2) Anastrozol }\end{array}$ & $1^{\mathrm{a}}$ linea & SLP & $\begin{array}{c}1: 13,8 \text { meses } \\
2: 11,1 \text { meses } \\
\&\end{array}$ & $\begin{array}{l}\text { 1: no alcanzada } \\
\text { 2: } 60,1 \text { meses }\end{array}$ \\
\hline CALGB $40302^{38}$ & 295 & $\begin{array}{l}\text { 1) Fulvestrant + lapatinib } \\
\qquad v s \\
\text { 2) Fulvestrant + placebo }\end{array}$ & $2^{\circ}$ línea & SLP & $\begin{array}{c}1: 4,7 \text { meses } \\
2: 3,8 \text { meses } \\
\&\end{array}$ & $\begin{array}{l}1: 30 \text { meses } \\
2: 26,4 \text { meses } \\
\&\end{array}$ \\
\hline
\end{tabular}

SLP: sobrevida libre de progresión SG: sobrevida global. *pacientes vírgenes a terapia endocrina. ${ }^{\star}$ diferencia no estadísticamente significativa.

HR 0,$81 ; 95 \%$ IC 0,65 a 1,$00 ; p=0,05)^{14}$. El análisis de subgrupos de este estudio sugirió que los beneficios de la combinación se restringían en gran medida a las mujeres que no habían recibido tamoxifeno adyuvante (vírgenes a TE). Nuevamente, características de la población incluida en los estudios podría explicar estos resultados contradictorios.

\section{Combinación de terapias endocrinas y moleculares en primera línea}

Primera línea de tratamiento en CMA la definiremos como aquella utilizada en pacientes con CMA que no han sido tratados previamente para su CMA. Mientras pueden haber recibido TE como parte del tratamiento inicial del CM (neoadyuvante, adyuvan- 
te), no han recibido TE para el CMA. La resistencia a TE la clasificaremos como:

- Resistencia de novo: Un paciente con enfermedad metastásica resistente al tratamiento (progresión antes de 6 meses de TE), o con recurrencia precoz después de terminar TE adyuvante (antes de 1-2 años del término de la TE adyuvante o intratratamiento).

- Resistencia adquirida o secundaria: aquella que se manifiesta como recurrencia del CM después de los 2 primeros años de TE adyuvante, o como la progresión de la enfermedad más de 6 meses después de la iniciación de TE en $\mathrm{CMA}^{4}$. Estas definiciones, aunque imperfectas y arbitrarias, han sido útiles en algunos ensayos clínicos para estratificar a pacientes y definir tipos de tratamiento ${ }^{15,16}$. En estudios clínicos recientes los criterios de inclusión han sido diferentes a estas definiciones clasificando a pacientes como aquellos con recaída antes o después del año. Evidencia preclínica sugiere que mecanismos de resistencia a la TE, como la vía PI3K/AKT/mTOR (fosfatidil inositol $3 \mathrm{kinasa} /$ proteína kinasa $\mathrm{B} / \mathrm{mammalian}$ target of rapamycin), alteraciones en la maquinaria del ciclo celular y la activación cruzada entre los receptores hormonales y la señalización de receptores de factores de crecimiento, entre otros, participarían de la resistencia a la $\mathrm{TE}^{15}$ (Figura 2). Revisaremos brevemente la evidencia clínica que sustenta el uso de terapias moleculares en uso hoy en CMA, las que están enfocadas, principalmente, en revertir los mecanismos de resistencia a la supresión estrogénica.

\section{Inhibidores de CDK4/6}

La proliferación celular autónoma, es una de las características de la célula neoplásica. La desregulación del ciclo celular subyace frecuentemente a esta ventaja comparativa. En $\mathrm{CM} \mathrm{RE}+$, particularmente de subtipo luminal B (Tabla 1) existe una desregulación de la expresión y función de ciclina $\mathrm{D}$, kinasas dependientes de ciclinas (CDK4/6) y de la proteína del retinoblastoma $(\mathrm{Rb})^{12}$ (Figura 2). El palbociclib (PD-0332991) es un inhibidor oral de CDK4/6 con evidencia preclínica de actividad inhibidora del crecimiento en células de CM RE + y sinérgico a

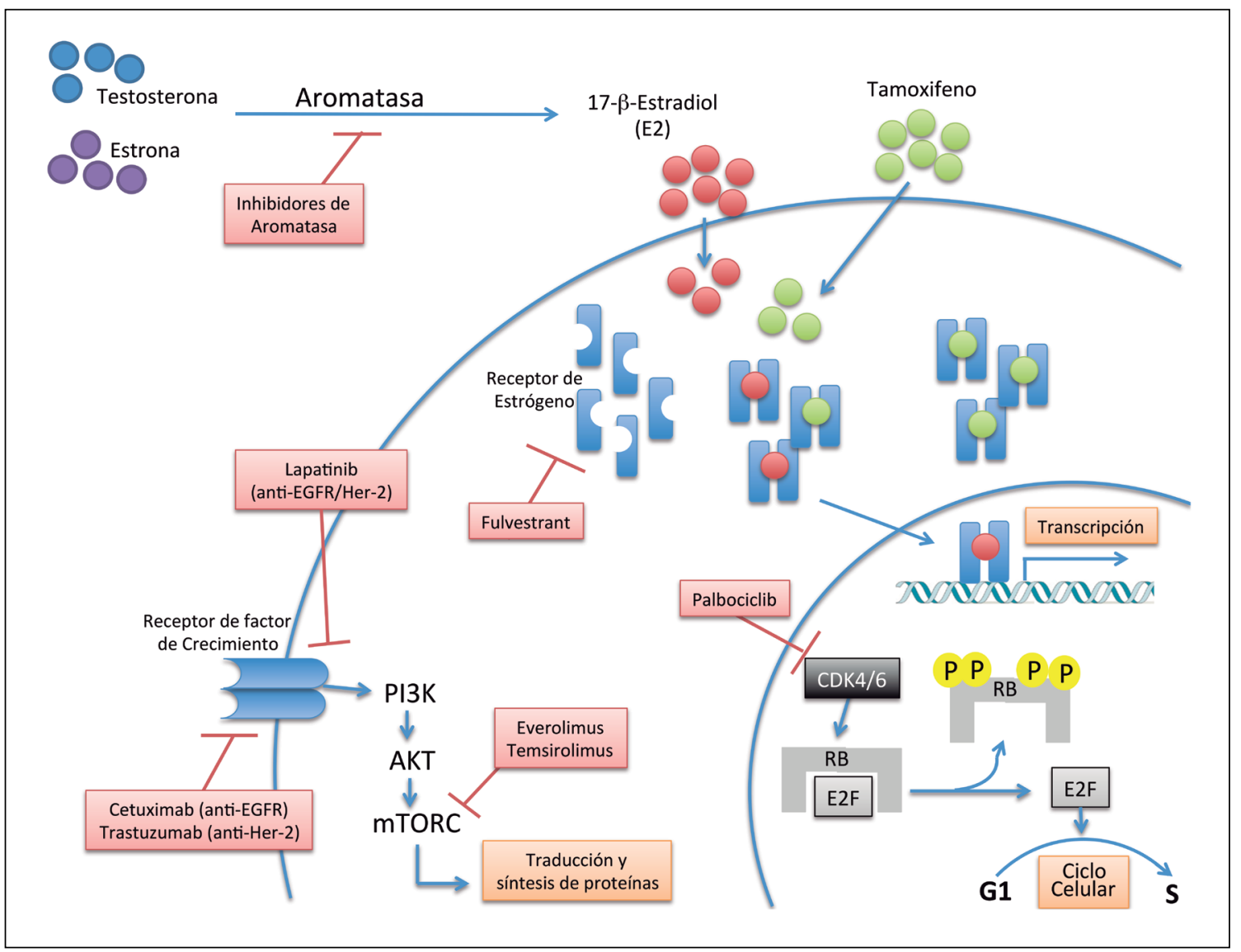

Figura 2. 
la supresión estrogénica ${ }^{17,18}$. En el estudio fase III Paloma $2^{18}$ realizado en mujeres con CMA RE+/ HER2 negativo, sin tratamiento sistémico previo para CMA, la adición de palbociclib al letrozol, en esta población muy sensible a estímulo endocrino, mejoró significativamente la SLP de 24,8 meses para la combinación versus 14,5 meses para el IA solo (HR 0,58; IC del 95\%: 0,46-0,72; p =0,001). Neutropenia ocurrió en el $66,4 \%$ de los pacientes que recibieron palbociclib, y $1,8 \%$ cursaron con neutropenia febril. Abemaciclib ${ }^{19}$ (LY2853219) y ribociclib (LEE011) ${ }^{20}$ son inhibidores adicionales de CDK4/6 que muestran igualmente una mejoría en la SLP, especialmente en pacientes con CMA RE+ (Tabla 4).

\section{Tratamiento de segunda línea}

Invariablemente todos los pacientes requerirán una nueva estrategia de tratamiento tras la aparición de resistencia primaria o secundaria.

Actualmente no se dispone de biomarcadores predictivos en la práctica clínica, para seleccionar el mejor tratamiento hormonal para CMA RE+ con resistencia a TE, y aún son factores clínicos los que guían el tratamiento (Figura 1) ${ }^{4,21}$. Después del fracaso del IA, el tamoxifeno es de beneficio clínico en aproximadamente el $50 \%$ de los casos, esto significa mejoría de los síntomas y estabilidad de la enfermedad; sin embargo, sólo un 10\% o menos de las pacientes logra una respuesta objetiva ${ }^{22}$. En el estudio TAMRAD, un estudio Fase II, los pacientes tratados con tamoxifeno después de una falla de IA tuvieron una SLP de 5,4 meses ${ }^{23}$. El estudio CONFIRM fue un estudio multicéntrico, doble ciego, de fase III, que incluyó 736 mujeres posmenopáusicas con CMA RE+, con progresión tras tamoxifeno o IA $^{24}$ y comparó diferentes dosis de fulvestrant. Los resultados mostraron que fulvestrant $500 \mathrm{mg}$ se asoció a mejor SLP (6,5 meses frente a 5,5 meses; $\mathrm{HR}=0,80$; IC del 95\%: 0,68; 0,94; $\mathrm{p}=0,006) \mathrm{y}$ SG (26,4 meses versus 22,3 meses; HR 0,81: IC del $95 \% 069-0,96 ; p=0,02)$. El beneficio sugerido en términos de $\mathrm{SG}$ en este estudio es único, dado que muy pocos tratamientos han demostrado mejoras en la SG de pacientes con CMA RE+ en estudios randomizados. La Tabla 4 muestra ensayos seleccionados de TE de segunda línea. Cabe destacar que mientras tenemos abundante información sobre el perfil molecular del CM primario, disponemos de muy poca información sobre los cambios moleculares que caracterizan o conducen a la enfermedad recurrente ${ }^{3}$. La mayoría de los ensayos clínicos no han sido capaces de recopilar o analizar de forma efectiva datos o tejidos que informen sobre mecanismos de resistencia específicos en un paciente individual los que podrían potencialmente ayudarnos a seleccionar la terapia posterior más apropiada.

\section{Inhibidores de PIK3CA / AKT / mTOR}

Las alteraciones en la vía de PI3K son las alteraciones moleculares más frecuentes en el CM RE+, identificadas en el $45 \%$ y el $29 \%$ de los tumores luminales A y B, respectivamente. Otros componentes de esta vía como son AKT y mTOR también se alteran con frecuencia ${ }^{12}$. La serina-treonina quinasa mTOR (del inglés mammalian target of rapamycina) regula una serie de procesos metabólicos en las células que integran el crecimiento celular, la proliferación celular, la supervivencia y las señales de motilidad mediadas por RE, HER2 y otros receptores de tirosina quinasa ${ }^{12}$. El BOLERO2, un estudio randomizado fase III, que comparó el everolimus (inhibidor de mTOR) y exemestano (un IA) versus exemestano y placebo en 724 pacientes con CMA RE+ con recurrencia o progresión mientras recibían o dentro de los 12 meses de completar un tratamiento con IA no esteroidal en adyuvancia ${ }^{25}$, o que progresan durante la terapia para la enfermedad metastásica resistentes a la TE, mostró una mejoría significativa en la SLP de 10,6 meses y 4,1 meses, respectivamente (HR, 0,36; 95\% IC 0,27 a 0,47 ; $\mathrm{p}<0,001)$, sin mejoría estadística en la SG. La adición de everolimus se asoció a mayor toxicidad con una proporción significativamente mayor de discontinuaciones y efectos adversos grado 3/4 tales como estomatitis, neumonitis no infecciosa e hiperglicemia, que usualmente no se ven con un IA como agente único. La identificación de biomarcadores predictivos para la inhibición de PI3K/mTOR sigue siendo una necesidad insatisfecha ${ }^{21}$. Tras el desarrollo de los inhibidores de mTOR, se están evaluando fármacos dirigidos a otros componentes de la vía que incluyen inhibidores de AKT y PI3KA, así como inhibidores de quinasa duales dirigidos tanto a mTOR como a PI3KA ${ }^{26}$.

\section{Combinación de terapia endocrina con inhibidores de CDK4/6}

Como se ha comentado anteriormente, los inhibidores de CDK4/6 parecen modular la TE. El reciente estudio PALOMA3 demostró que el palbociclib se asocia con un beneficio significativo en los pacientes con CMA RE+, previamente tratados. Este estudio doble ciego, aleatorizó a 521 pacientes con CMA RE+/HER2 negativo con progresión tras TE, para recibir palbociclib y fulvestrant o placebo 
y fulvestrant. El análisis interino de este estudio demostró la eficacia de la combinación con una mediana de SLP de 9,2 meses con palbociclib más fulvestrant y 3,8 meses con fulvestrant (HR 0,42; IC del 95\%: 0,32 a 0,56; $\mathrm{p}<0,001)^{17}$. Aun no existen datos acerca del beneficio en SG. Desafortunadamente, a pesar del esfuerzo inicial en el estudio PALOMA1 al intentar identificar una población molecularmente seleccionada de pacientes (amplificación de ciclina D1 o pérdida de p16), hasta ahora no se ha identificado la población de pacientes que más se beneficiaría de la inhibición de la CDK4/6 ${ }^{27}$.

\section{Otras estrategias}

La modulación epigenética de la expresión génica ha sido abordada en pacientes con CMA RE $+{ }^{12}$. La resistencia a IA se caracteriza por un crecimiento independiente de estrógeno y los mecanismos de resistencia pueden incluir disminución de la expresión del RE. Entinostat, un inhibidor selectivo de la histona desacetilasa (HDAC), conduce al aumento de la expresión del RE y la aromatasa en los estudios preclínicos y se están esperando los resultados de estudios clínicos ${ }^{28}$.

Además, de los IA clásicos antes mencionados, SERDs y MSRE, otras estrategias terapéuticas en desarrollo incluyen la antiangiogénesis, la inhibición de mTOR, la inhibición de CDK4/6 y la modulación epigenética abordada en nuestra discusión, el papel de los antiandrógenos, evasión de apoptosis, las interacciones con el microambiente tumoral, mutaciones de ESR $1^{12,29}$, así como otros inhibidores de la tirosina quinasa (TKIs) y vías de señalización de factores de crecimiento ${ }^{15}$.

Varias alternativas de TE evaluadas muchos años atrás, aún son opciones a considerar en el paciente que progresa y persiste con evidencia clínica de sensibilidad endocrina. Los progestágenos (acetato de megestrol, medroxiprogesterona) tienen un nivel de respuesta del $25 \%$, pero se asocian con aumento de peso, retención de líquidos y eventos tromboembóli$\cos ^{4}$. Los andrógenos (testosterona, fluoximesterona, danazol) se usan raramente, pero han reportado respuestas positivas, aunque asociadas con eventos adversos significativos

Los estrógenos (dietilestilbestrol, etinilestradiol) se han utilizado paradójicamente para tratar CMA $\mathrm{RE}+$ mostrando una eficacia similar al tamoxifeno, pero una mayor toxicidad ${ }^{30}$. Estudios recientes sugieren que la terapia con estrógenos puede ser eficaz en tumores con amplificación de $E S R 1^{29}$. A pesar del bajo número de pacientes estudiados y la falta de datos prospectivos aleatorizados, la discontinuación de la TE y observación, como se práctica selectivamente en el cáncer de próstata, es otra estrategia potencial para los pacientes con $\mathrm{CMA} \mathrm{RE}+{ }^{4}$.

\section{Responsabilidades éticas}

Protección de personas y animales. Los autores declaran que para esta investigación no se han realizado experimentos en seres humanos ni en animales.

Confidencialidad de los datos. Los autores declaran que en este artículo no aparecen datos de pacientes.

Derecho a la privacidad y consentimiento informado. Los autores declaran que en este artículo no aparecen datos de pacientes.

\section{Conflicto de Intereses}

La Dra. Pérez-Sepúlveda recibió apoyo para la investigación de Pfizer. Todos los otros autores declaran no tener conflicto de intereses.

\section{Referencias}

1. Ferlay J, Soerjomataram I, Ervik M, Dikshit R, Eser S, Mathers C, et al. GLOBOCAN 2012 v1.0, Cancer Incidence and Mortality Worldwide: IARC CancerBase No. 11. 2012. p. Lyon, France: International Agency for Research on.

2. www.deis.cl. Estadísticas de natalidad y mortalidad. [revisado el 14 de de abril de 2017]. Disponible en: http://www.deis.cl/ estadisticas-de-natalidad-y-mortalidad/
3. Sánchez C, Camus M, Medina L, Oddo D, Artigas R, Pérez Sepúlveda A, et al. Clinico-Pathologic Subtypes of Breast Cancer Primary Tumors Are Related to Prognosis after Recurrence. Asian Pac J Cancer Prev. 2016;17:5081-6.

4. Cardoso F, Costa A, Senkus E, Aapro $\mathrm{M}$, Andre F, Barrios CH, et al. 3rd ESO-ESMO International Consensus Guidelines for Advanced Breast Cancer ( ABC 3 ) Special article. Ann Oncol 2017;28:16-33.

5. Wilcken N, Hornbuckle J, Ghersi D.
Chemotherapy alone versus endocrine therapy alone for metastatic breast cancer. Cochrane Database of Systematic Reviews 2003;2:CD002747.

6. Barrios C, Forbes JF, Jonat W, Conte P, Gradishar W, Buzdar A, et al. The sequential use of endocrine treatment for advanced breast cancer: Where are we? Ann Oncol. 2012;23:1378-86.

7. Gradishar WJ. Tamoxifen-What Next? Oncologist 2004;9:378-84.

8. Beresford M, Tumur I, Chakrabarti J, Barden J, Rao N, Makris A. A qualitative 
systematic review of the evidence base for non-cross-resistance between steroidal and non-steroidal aromatase inhibitors in metastatic breast cancer. Clin Oncol (R Coll Radiol) 2011;23:209-15.

9. Mauri D, Pavlidis N, Polyzos NP, Ioannidis JPA. Survival With Aromatase Inhibitors and Inactivators Versus. J Natl Cancer Inst 2017;98:1285-91.

10. Ellis MJ, Llombart-Cussac A, Feltl D, Dewar JA, Jasiówka M, Hewson N, et al. Fulvestrant $500 \mathrm{mg}$ Versus Anastrozole $1 \mathrm{mg}$ for the First-Line Treatment of Advanced Breast Cancer: Overall Survival Analysis From the Phase II FIRST Study. J Clin Oncol 2015;33:3781-7.

11. Robertson JFR, Bondarenko IM, Trishkina E, Dvorkin M, Panasci L, Manikhas A, et al. Fulvestrant $500 \mathrm{mg}$ versus anastrozole $1 \mathrm{mg}$ for hormone receptor-positive advanced breast cancer (FALCON): an international, randomised, double-blind, phase 3 trial. Lancet 2017;388:2997-3005.

12. Ma CX, Reinert T, Chmielewska I, Ellis MJ. Mechanisms of aromatase inhibitor resistance. Nat Rev Cancer 2015;15:26175 .

13. Bergh J, Jo P, Lidbrink EK, Trudeau M, Eiermann W, Brattstro D. FACT: An Open-Label Randomized Phase III Study of Fulvestrant and Anastrozole in Combination Compared With Anastrozole Alone As First-Line Therapy for Patients With Receptor-Positive Postmenopausal Breast Cancer. J Clin Oncol. 2017;30:1919-25.

14. Livingston RB, Hortobagyi GN. Combination Anastrozole and Fulvestrant in Metastatic Breast Cancer. N Engl J Med. 2012;367:435-44.

15. Miller TW. Endocrine resistance: what do we know? Am Soc Clin Oncol Educ book 2013;33:e37-e42.

16. Wilson S, Chia SK. Treatment algorithms for hormone receptor-positive advanced breast cancer: applying the results from recent clinical trials into daily practiceinsights, limitations, and moving forward. Am Soc Clin Oncol Educ Book 2013;33:e20-e7.

17. Turner NC, Ro J, Andre F, Loi S, Verma $\mathrm{S}$, Iwata $\mathrm{H}$, et al. Palbociclib in HormoneReceptor-Positive Advanced Breast Cancer. N Engl J Med. 2015;373:209-19.

18. Finn RS, Martin M, Rugo HS, Jones S,
Im S-A, Gelmon K, et al. Palbociclib and Letrozole in Advanced Breast Cancer. N Engl J Med. 2016;375:1925-36.

19. Sledge GW, Toi M, Neven P, Sohn J, Inoue K, Pivot X, et al. MONARCH 2: Abemaciclib in Combination With Fulvestrant in Women With HR +/ HER2-Advanced Breast Cancer Who Had Progressed While Receiving Endocrine Therapy. J Clin Oncol. 2017;35:2875-84.

20. Hortobagyi GN, Stemmer SM, Burris HA, Yap Y-S, Sonke GS, Paluch-Shimon S, et al. Ribociclib as First-Line Therapy for HR-Positive, Advanced Breast Cancer. N Engl J Med. 2016;375:1738-48.

21. Rugo HS, Rumble RB, Macrae E, Barton DL, Connolly HK, Dickler MN, et al. Endocrine Therapy for Hormone Receptor-Positive Metastatic Breast Cancer: American Society of Clinical Oncology Guideline. J Clin Oncol 2016;34:3069-103.

22. Reinert $\mathrm{T}$, Barrios $\mathrm{CH}$. Optimal management of hormone receptor positive metastatic breast cancer in 2016. Ther Adv Med Oncol 2015;7:304-20.

23. Bachelot T, Cropet C, Ray-coquard I, Ferrero J, Freyer G, Abadie-lacourtoisie S, et al. Randomized Phase II Trial of Everolimus in Combination With Tamoxifen in Patients With Hormone Receptor-Positive, Human Epidermal Growth Factor Receptor 2-Negative Metastatic Breast Cancer With Prior Exposure to Aromatase. J Clin Oncol 2017;30:2718-24.

24. Leo A Di, Jerusalem G, Petruzelka L, Torres R, Bondarenko IN, Khasanov R, et al. Final overall survival: Fulvestrant 500 $\mathrm{mg}$ vs $250 \mathrm{mg}$ in the randomized confirm trial. J Natl Cancer Inst 2014;106:djt337.

25. Piccart M, Hortobagyi GN, Campone M, Pritchard KI, Lebrun F, Ito Y, et al. Everolimus plus exemestane for hormonereceptor-positive, human epidermal growth factor receptor-2-negative advanced breast cancer: overall survival results from BOLERO-2. Ann Oncol. 2014;25:2357-62.

26. Sanchez CG, Ma CX, Crowder RJ, Guintoli T, Phommaly C, Gao F, et al. Preclinical modeling of combined phosphatidylinositol-3-kinase inhibition with endocrine therapy for estrogen receptor-positive breast cancer. Breast Cancer Res. 2011;13:R21.
27. Finn RS, Crown JP, Ettl J, Schmidt M, Bondarenko IM, Lang I, et al. Efficacy and safety of palbociclib in combination with letrozole as first-line treatment of ER-positive, HER2-negative, advanced breast cancer: expanded analyses of subgroups from the randomized pivotal trial PALOMA-1/TRIO-18. Breast Cancer Res. 2016;18:67.

28. Falahi F, van Kruchten M, Martinet N, Hospers GAP, Rots MG. Current and upcoming approaches to exploit the reversibility of epigenetic mutations in breast cancer. Breast Cancer Res. 2014;16:412.

29. Li S, Shen D, Shao J, Crowder R, Liu W, Prat A, et al. EndocrineTherapy-Resistant ESR1 Variants Revealed by Genomic Characterization of Breast-Cancer-Derived Xenografts. Cell Rep 2013;4:1116-30.

30. Ellis MJ, Gao F, Dehdashti F, Jeffe DB, Marcom PK, Carey LA, et al. Lowerdose $v s$ high-dose oral estradiol therapy of hormone receptor-positive, aromatase inhibitor-resistant advanced breast cancer: a phase 2 randomized study. JAMA 2009;302:774-80.

31. Johnston SR, Kilburn LS, Ellis P, Dodwell D, Cameron D, Hayward L, et al. Fulvestrant plus anastrozole or placebo versus exemestane alone after progression on non-steroidal aromatase inhibitors in postmenopausal patients with hormone-receptor-positive locally advanced or metastatic breast cancer (SoFEA): a composite, multicentr. Lancet Oncol. 2013;14:989-98.

32. Yardley DA, Noguchi S, Pritchard KI, Burris HA 3rd, Baselga J, Gnant M, et al. Everolimus plus exemestane in postmenopausal patients with $\mathrm{HR}(+)$ breast cancer: BOLERO-2 final progression-free survival analysis. Adv Ther. 2013;30:870-84.

33. Cristofanilli M, Turner NC, Bondarenko I, Ro J, Im S-A, Masuda N, et al. Fulvestrant plus palbociclib versus fulvestrant plus placebo for treatment of hormonereceptor-positive, HER2-negative metastatic breast cancer that progressed on previous endocrine therapy (PALOMA-3): final analysis of the multicentre, double-blind, phas. Lancet Oncol. 2016;17:425-39. 
34. Noguchi S, Kim HJ, Jesena A, Parmar V, Sato N, Wang H-C, et al. Phase 3, open-label, randomized study comparing 3-monthly with monthly goserelin in pre-menopausal women with estrogen receptor-positive advanced breast cancer. Breast Cancer 2016;23:771-9.

35. Iwata H, Masuda N, Ohno S, Rai Y, Sato Y, Ohsumi S, et al. A randomized, doubleblind, controlled study of exemestane versus anastrozole for the first-line treatment of postmenopausal Japanese women with hormone-receptor-positive advanced breast cancer. Breast Cancer Res
Treat 2013;139:441-51.

36. Wolff AC, Lazar AA, Bondarenko I, Garin AM, Brincat S, Chow L, et al. Randomized phase III placebo-controlled trial of letrozole plus oral temsirolimus as first-line endocrine therapy in postmenopausal women with locally advanced or metastatic breast cancer. J Clin Oncol. 2013;31:195-202.

37. Dickler MN, Barry WT, Cirrincione CT, Ellis MJ, Moynahan ME, Innocenti F, et al. Phase III Trial Evaluating Letrozole As First-Line Endocrine Therapy With or Without Bevacizumab for the Treatment of Postmenopausal Women With Hormone Receptor-Positive Advanced-Stage Breast Cancer: CALGB 40503 (Alliance). J Clin Oncol. 2016;34:2602-9.

38. Burstein HJ, Cirrincione CT, Barry WT, Chew HK, Tolaney SM, Lake DE, et al. Endocrine Therapy With or Without Inhibition of Epidermal Growth Factor Receptor and Human Epidermal Growth Factor Receptor 2: A Randomized, Double-Blind, Placebo-Controlled Phase III Trial of Fulvestrant With or Without Lapatinib for Postmenopausal Women. J Clin Oncol. 2014 10;32:3959-66. 